\title{
Two Separate Tumor Masses Present in the Same Organ
}

National Cancer Institute

\section{Source}

National Cancer Institute. Two Separate Tumor Masses Present in the Same Organ. NCI

Thesaurus. Code C154521.

A finding indicating the presence of two separate tumor masses in the same organ. 